\title{
Illusions comiques et illusions optiques : l'œil baroque
}

\section{Theatrical and Optical Illusions: The Baroque Eye}

JeAn-Claude Vuillemin [jcvuillemin@psu.edu]

The Pennsylvania State University, États-Unis d'Amérique

\section{RÉSUMÉ}

$\mathrm{Si}$, comme le prétend Foucault, l'épistémè « classique », enferma les corps, la première modernité présida à cet autre enfermement généralement passé sous silence : l'asservissement de l'œil de chair à l'œil de l'esprit. Pour que voir renoue avec savoir, le second devra désormais primer sur le premier. L'on estime se doter ainsi d'une vision qui va au-delà du visible; d'un voir qui excède la vue. À partir des travaux pionniers de ces « fondateurs de discursivité » (Foucault) que furent Kepler et Descartes, cet article retrace la généalogie de l'assujettissement de l'œil organique à la réflexion analytique du regard raisonnable et montre son illustration sur la scène baroque contemporaine.

\section{MotS-CLÉS}

Illusion ; vision ; optique ; science moderne ; théâtre baroque ; Descartes ; Kepler

\begin{abstract}
If, as Foucault alleged, the so-called "classical episteme" incarcerated the bodies, early modernity presided to another type of imprisonment he does not mentioned: the enslavement of the physical eyeball to the eye of the mind. In order for seeing to regain its compatibility with knowing, the latter eye will have henceforth to prevail over the former. Grounded in Kepler and Descartes' pioneer writings related to $17^{\text {th }}$-century eyesight issues, this article depicts the genealogy of this too-often ignored enslavement and shows its manifestation on the contemporary baroque stage.
\end{abstract}

\section{KEYWORDS}

Illusion; vision; optics; modern science; baroque drama; Descartes; Kepler

REÇU 2019-03-18 ; ACCEPTE 2019-06-12 
"Pauvres yeux qui ne sentez rien, vous n'êtes pas le meilleur du corps. »

P. Quignard, Performances de ténèbres, p. 90.

« N’apprendras-tu jamais, âme basse, et grossière, À voir par d'autres yeux que les yeux du vulgaire?»

P. Corneille, Rodogune, II, 2.

Nonobstant le caractère parfois péremptoire de ses assertions, Foucault a judicieusement caractérisé cette épistémè qu'il qualifie inopportunément de "classique ${ }^{1}$ » en privilégiant l'écart entre le signe et ce qu'il représente et en insistant sur la rupture des paradigmes épistémologiques qui, jusqu'alors, étaient parvenus à lier, en un tout cohérent, l'univers, le monde et l'individu (Foucault 1966 : 32-86). Il y eut faillite de ce système harmonieux lorsque, dénouant son appartenance au savoir, la ressemblance alla même jusqu'à l'entraver considérablement. Ainsi que le prouvera définitivement La Dioptrique de Descartes (1637), contemporaine de L'Illusion comique de Corneille (1636), du monde à l'œil et, surtout, de l'œil au cerveau, rien n'est plus transmis qui se ressemble. Comme en témoignent maints épisodes de la scène baroque, la ressemblance, loin d'être une caution de vérité, est au contraire une source d'illusion, une porte ouverte à l'erreur. Cela à une époque qui, remarque Foucault, est « le temps privilégié du trompe-l'œil, de l'illusion comique, du théâtre qui se dédouble [...] ; c'est le temps des sens trompeurs » (Foucault 1966: 65). Certainement. Et si l'on accepte l'idée que l'épistémè est, comme l'avance Foucault, une espèce d'«a priori historique» (Foucault 1969: 166 sqq.) qui, à une époque donnée, suscite la production et la transformation des savoirs, rien de très surprenant à ce que des similitudes existent entre des domaines apparemment étrangers l'un à l'autre. C'est l'une de ces parentés négligées que cet article se propose de mettre au jour : celle qui unit la mutation épistémologique du regard à un motif récurrent du théâtre de la première modernité. Ou, pour le dire autrement, je souhaiterais montrer que certaines révélations optiques de la science nouvelle trouvent une parfaite illustration dans le théâtre baroque qui leur est contemporain.

Les protagonistes de ces illusions comiques, i. e. dramatiques, s'avèrent souvent incapables de distinguer le vrai du faux et cette inaptitude procède bien évidemment de multiples sources. Toutefois, c'est généralement pour avoir fait trop rapidement confiance au rapport de leurs yeux, "Ces astres qui pourraient en imposer aux Dieux » (Rotrou, Antigone, I, 4), qu'ils ou elles sont trompés. Et les fréquentes pétitions de principe qui, telle celle de Lydie, cet autre personnage de Rotrou : «À des yeux clairvoyants la feinte est inutile » (La Sour, I, 1), ne changent rien à l'affaire. En fait, sitôt proférées, ces proclamations optimistes se trouvent invariablement infirmées.

1 Rétif aux périodisations totalitaires et attentif aux discursivités qui les véhiculent, Foucault a néanmoins accepté comme allant de soi la notion de "classicisme ». Alors que le premier terme du syntagme "épistémè classique " est abondamment discuté, le second terme ne l'est jamais et est accepté comme allant de soi. 
"Pour moi, je ne sais point tant de philosophie », se targue à son tour le Gros-René du Dépit amoureux de Molière : "Ce que voyent mes yeux, franchement je m’y fie » (I, 1). Mais, comme tous ceux et celles qui font trop rapidement confiance « au rapport de [leurs] yeux » (V, 7), l'obtus valet d'Éraste se montre tout à fait incapable de démêler les imbroglios et les jeux d'illusion dans lesquels lui et son maître évoluent.

Ce n'est pas sans un malin plaisir, semble-t-il, que les dramaturges de l'époque mettent constamment en scène l'inanité du regard abusé d'individus persistant à affirmer le bien fondé d'une modalité d'interprétation du monde qui assigne imprudemment l'ordre du savoir à l'ordre du voir. C’est d'ailleurs sur ce parti pris épistémologique - très proche du règne médiatique actuel définissant comme existant ce qui est montré - que miseront les manipulateurs des apparences, ceux du théâtre baroque comme les habiles de nos médias modernes, pour tâcher de réorganiser à leur profit la scène équivoque du monde. On peut certes faire comprendre quelque chose par l'argumentation déductive d'un raisonnement abstrait, mais il est sans doute bien plus économique, hier comme aujourd'hui, de faire voir pour faire croire ; de convaincre par les sens plutôt que de persuader par le sens. Telle est en tout cas la stratégie d'Alcandre-Corneille, le magicien-dramaturge de L'Illusion comique qui, afin de faire l'apologie du théâtre, " amour de tous les bons esprits [...] fief dont les rentes sont bonnes » $(\mathrm{V}, 6)$, commence par satisfaire les sens de son public avant de s'adresser à sa raison; de montrer afin de démontrer. Et la force de cette magistrale plaidoirie en acte réside tout entière dans la mystification dont Pridamant et les véritables spectateurs de la pièce, dont Pridamant est l'analogon scénique, ont été la cible. Contrairement pourtant à ce qui se passe dans L'Illusion comique, les mystifications mises en scène dans le théâtre baroque se déroulent en général sous les yeux censément " clairvoyants » d'un public dont l'agrément résulte autant de voir des personnages trompés que d'être dans le secret des stratagèmes de la tromperie. Il est d'ailleurs habituel qu'un personnage témoin de la mystification en cours fasse un commentaire sur la nature théâtrale de celle-ci et oblige de la sorte l'instance spectatrice à ne pas perdre de vue le caractère illusoire de ce qui se déroule sur la scène. En attirant ainsi le regard du spectateur sur ce que la convention théâtrale s'évertue généralement à oblitérer, ce retour réflexif du théâtre sur lui-même a quelque chose d'insolite, voire d'insolent. Lié à la rupture de l'illusion dramatique, cette « douce illusion » qui est, selon La Bruyère, " tout le plaisir du théâtre » (La Bruyère 2011 : 92), le charme de cette distanciation brechtienne avant la lettre enchante en désenchantant. Mais, tout en soulignant le processus de signification sémiotique, ces fréquentes exhibitions du code théâtral ne font pas qu'entraver le pacte de fascination requis par l'illusion mimétique au profit d'une réflexion sur le mécanisme de l'illusion comique, elles débouchent également sur une réflexion épistémologique du regard.

Préservés des erreurs d'interprétation qui affectent les personnages-trompés, les spectateurs bénéficient ordinairement d'un savoir identique à celui des personnages-trompeurs dont l'astuce primordiale consiste principalement à donner à voir pour décevoir. Quatre pièces enfreignent toutefois cette norme en mystifiant les spectateurs eux-mêmes. Outre L'Illusion comique de Corneille (1636), il s'agit des deux Comédie des comédiens de Gougenot et de Scudéry (1633) et, vingt ans plus tard, du Véritable Saint Genest de Rotrou $(1645)^{2}$. Dans ces pièces, l'instance spectatrice

2 Pour une analyse du mécanisme de l'illusion dans ces pièces et des modalités de la structure en abyme de «théâtre dans le théâtre ", voir Vuillemin 2001. 
ne doit pas se contenter d'entériner une dichotomie entre illusion et réalité en entretenant un lien de complicité avec les personnages-illusionnistes, elle est contrainte de mettre en cause la validité même de son propre regard. D’où le caractère extrêmement malicieux et profondément ironique de l'exhortation d'Alcandre-Corneille : "N'en croyez que vos yeux " (L'Illusion comique, $\mathrm{V}, 6$ ), à la fin d'une pièce qui vient admirablement, et mieux que toutes autres, de prouver de façon irréfutable que le regard non prévenu est pernicieux et qu'il ne débouche que sur une profonde méprise.

Comme dans l'anamorphose, le secret de L'Illusion comique est là, visible, mais pour le discerner il eût été nécessaire de se déplacer. Pris au piège du visible des anamorphoses picturales comme il l'est à celui de ces représentations théâtrales qui l'amusent et l'abusent, l'œil se laisse facilement obnubiler par ce qui se présente sur l'axe de la visibilité immédiate. Pourtant, sur la toile comme sur la scène, le visible, qui passe pour vrai à l'œil, est illusion. Au théâtre, comme dans l'anamorphose ou dans la cosmographie, il y a le monde visible, mais erroné : dans L'Illusion comique, c'est Clindor, trucidé par un mari jaloux, c'est la tache informe au premier plan des Ambassadeurs d'Holbein, c'est le soleil tournant apparemment autour de la terre immobile ; puis il y a l'invisible, mais vrai : Clindor, comédien d'une troupe théâtrale interprétant un rôle tragique, c'est l'image d'un crane humain, symbole caractéristique des peintures dites de "Vanités", que révèle le tableau d'Holbein perçu du bon point de vue, ou encore ce que prouve, contre l'autorité des sens, d'Aristote et de la Bible, la nouvelle cosmographie : mobile, la terre tourne bel et bien autour du soleil, immobile. Exemples paradigmatiques qui invitent à se méfier au plus haut point de l'observation candide et/ou naïve du quotidien et de l'expérience apparemment indubitable des sens. Images du monde du théâtre autant que métaphores du théâtre du monde, L'Illusion comique, les deux Comédie des comédiens et le Saint Genest, sont également des allégories de l'illusion optique.

Alors que depuis Aristote, le « désir de connaître » était anthropologiquement fondé, puisque lié au «plaisir causé par les sensations » et, précisait l'auteur de la Métaphysique, "plus que toutes les autres, [par] les sensations visuelles » (Aristote 1991 : 2), le théâtre baroque dans son ensemble ne cesse de mettre en lumière que la connaissance est incompatible avec la séduction des sens ; que l'évidence sensible, et surtout visible, n'est plus garante du vrai : elle est au contraire responsable d'une infinité d'erreurs et de méprises. Et, lorsqu'elle n'est pas une puissance formidable d'illusion, la vue n'est jamais un outil efficace de connaissance ${ }^{3}$. Elle n'est, au mieux, qu'un simple support de reconnaissance dont la manifestation la plus ordinaire est le regrettable réflexe de ramener sans cesse l'inconnu au connu ${ }^{4}$. C'est ce qu'illustrent plusieurs épisodes de la scène baroque, et notamment du théâtre de Rotrou (Vuillemin 2007), où il est courant que les personnages soient victimes du regard spontané qu'ils portent sur le monde. Un regard qui correspond à cette manière de voir impressionniste et fallacieuse que, dans sa lettre à François Sublet de Noyers (vers 1641), Poussin appelle aspect et qu'il oppose au prospect, c'est-à-dire à

3 Ainsi, si l'on pense aux nombreuses scènes théâtrales où il est question d'une prétendue « voix secrète » du sang, on constate que celle-ci est tout à fait inaudible pour le regard et quelle ne se fait finalement entendre qu'après que l'identité du personnage a été dûment établie.

4 Selon William Shea, c'est parce qu'il connaissait l'hypothèse émise par certains savants de l'Antiquité selon laquelle la Lune était une planète comparable à la Terre, que Galilée aurait été en mesure de voir ce qu'il a vu, sans cela, " he would not have seen mountains and valleys when he focused his primitive telescope on our satellite » (Shea 2000 : 99). 
l'acte intellectuel relevant d'un " office de raison ${ }^{5}$ " au terme duquel on obtient un intuitus sur la forme et les propriétés spatiales réelles de l'objet. Sur la toile comme sur la petite scène du théâtre ou sur la grande scène du monde, il est démontré qu'une vision adéquate au visible ne saurait se satisfaire d'un processus purement charnel : elle requiert le passage de l'œil fugace et instinctif de la perception au regard raisonné et raisonnable de l'observation. Cette manière de considérer le regard est en tout point conforme à la nouvelle conception de la vision telle que la conçoit à la même époque la science moderne.

Même si certains modes de pensée antérieurs à ceux de la science moderne persistent, et s'il est plus que probable que les dramaturges n'aient eu, au mieux, qu'une connaissance superficielle des subtilités scientifiques élaborées dans les cercles savants, il n’en reste pas moins que plusieurs péripéties de leurs intrigues théâtrales peuvent se lire comme une propédeutique à la nouvelle connaissance optique par l'intermédiaire de la mise au jour des arcanes de l'illusion comique. Mettant en scène le passage d'une vision aveugle à un regard clairvoyant, ces épisodes attestent que la validité gnoséologique du regard n’est pas donnée, qu'elle doit être savamment construite et raisonnée.

Sans mettre en question la prévalence de la vision sur les autres sens, la première modernité se montra en effet extrêmement dubitative quant à sa présumée capacité à donner une transcription fidèle du réel. Dans le domaine de la représentation, c'est ce qu'illustre, par exemple, l'avènement de la perspective, dont Rotrou souligne l'artifice dans une scène de Saint Genest (II, 1). S’il est indéniable que la perspective, fruit du désir de réalisme des décennies précédentes, constitue une avancée notable dans le champ de la représentation, c'est en dérogeant au principe de similitude qui, jusqu’alors, avait été la pierre de touche de la vérité. Capable de simuler les causes de la vision en produisant dans l'œil le même effet que la chose vue, et non en essayant de reproduire scrupuleusement celle-ci, la perspectiva artificialis, par le biais d'une technique illusionniste la rapprochant des trompe-l'œil dont l'époque était friande, offre une représentation indifférente à la ressemblance. Ce faisant, la perspective rompait avec l'idée traditionnelle de la vision qui, notamment à travers la notion polysémique de species $^{6}$, postulait l'identité parfaite de la forme sensible dans la chose vue et dans l'être voyant.

De même, ce n'est pas parce que la vision bénéficia à l'époque d'avancées considérables dans le domaine technologique, voire littéraire, que la capacité cognitive du regard s'intensifia. Au contraire. Dans le même temps que le perfectionnement des instruments optiques donnait au regard les moyens de transcender les compétences visuelles communes et de repousser les limites du visible, l'avènement d'une littérature fabuleuse lui offrait aussi la possibilité de se prolonger dans les nombreux voyages cosmiques qui lui étaient proposés. À l'instar des «merveilleuses lunettes » qu'évoque Descartes dans l'incipit de La Dioptrique, ces fictions littéraires, auxquelles on pourrait encore ajouter les machines de théâtre et de l’opéra qui entraînaient l'esprit vers

5 Reprenant une distinction déjà faite par Alhazen et Vitellion, Poussin écrit qu' « il y a deux manières de voir les objets, l'une en les voyant simplement, et l'autre en les considérant avec attention. [...] on peut dire que le simple aspect est une opération naturelle, et que ce que je nomme le prospect est un office de raison qui dépend de trois choses, savoir de l'œil, du rayon visuel et de la distance de l'œil à l'objet » (Poussin $1964: 62-63$ ).

6 Le terme pouvait aussi bien s'appliquer « à ce qui émanait ponctuellement d'un objet, à ce qui se diffusait dans le milieu intermédiaire, et à l'image globale (ou à la forme) qui agissait sur le récepteur» (Pantin 2010 : 263). 
l'au-delà7, portaient la vision « beaucoup plus loin que n'avait coutume d'aller l'imagination de nos pères » (Descartes 1996 : 1, 81). Elles confirmaient ainsi ce que la science était en train de démontrer : l'individu est capable de voir au-delà de ce que lui procurent ses sens naturels. Transformant le regard, ces dispositifs artificiels vont donner aux Modernes de nouveaux yeux pour voir. Mais, alors que le dispositif perspectif suscite le soupçon que la profondeur apparente du monde n'est peut-être, comme les effets de relief du châssis peint de la pièce de Genest, qu'une illusion de surface plane, les télescopes et autres microscopes, à l'image des anamorphoses - ces transgressions de la «perfide Analogie » comme les considérera Barthes (Barthes 2002: 624) qui font voir ce que l'œil ne voit pas, permettent de pénétrer des surfaces que le regard ordinaire jugeait jusqu’alors infranchissables. La connaissance procédant de la vision deviendra dès lors extrêmement problématique puisque, à un accès prétendument direct et immédiat à la réalité, l'efficacité du regard dépendra, dans la science comme au théâtre, de la bonne interprétation des signes, voire de l'interprétation de signes créés par des instruments.

En outre, si le franchissement de l'évidence visuelle était certainement en mesure de stimuler le savoir, il pouvait aussi, paradoxalement, encourager le scepticisme en ce qu'il révélait l'impossibilité d'une connaissance assurée : plus les frontières du visible reculent, plus s'affirme l'existence de réalités encore invisibles. Ce qui est perçu a beau être considérablement augmenté, il s'avère néanmoins infime par rapport à ce qui, on ne saurait maintenant en douter, demeure encore inaperçu. Pire d'un point de vue épistémologique, si les avancées de la science nouvelle marquent un progrès indéniable dans la perception du visible et si elles établissent une proximité organique entre l'œil et l'instrument optique, elles soulèvent du même coup l'embarrassante question de savoir si, comme en ce qui concerne les mondes fantastiques que dévoile la littérature, les phénomènes révélés par l'instrument ne dépendent pas exclusivement de celui-ci. Puisque différents télescopes ou microscopes montrent des phénomènes distincts, il n'est pas déraisonnable de postuler qu'un œil autre percevrait un monde différent. L’argument rejoint le raisonnement sceptique qui connaît d'ailleurs à l'époque un regain d'intérêt et selon lequel il est impossible de se prononcer sur un monde qui, nous parvenant par l'entremise des sens, dépend de ces derniers et non de sa véritable nature. Telle était déjà l'opinion de Montaigne qui, après avoir repris à son compte l'affirmation de Sextus Empiricus quant à la carence sensitive de l'homme par rapport à l'animal, ne peut que logiquement conclure : « nous ne savons plus quelles sont les choses en vérité : car rien ne vient à nous que falsifié et altéré par nos sens » (Montaigne 2004 : 600).

Prolepse, en quelque sorte, au perspectivisme nietzschéen pour lequel non seulement les conditions physiques, mais aussi les dispositions éthiques, déterminent la perception, ce relativisme visuel rompt en tout cas avec la confiance aristotélicienne en la nature des choses que les sens étaient supposés communiquer. Il n'est certainement pas interdit de penser qu'entre les nouvelles conceptions optiques et la résurgence, dans la foulée de Montaigne, des courants sceptiques hérités de l'Antiquité il y eut, sinon convergence épistémologique, du moins affinité philosophique, comme il y a parenté entre illusions optiques et illusions comiques. Mais, alors que ces dernières sont aujourd'hui sorties des oubliettes où les avait maintenues la tradition, l'œil baroque, surtout chez les

7 À propos de la Finta Pazza que Mazarin fit représenter dans la salle du Petit Bourbon, La Gazette de Renaudot relate que l'artifice des machines de Torelli ne transporte « pas moins les yeux de l'esprit que ceux du corps par des mouvements imperceptibles » (16 déc. 1645). La Bruyère note que « la machine [...] augmente et embellit la fiction » des opéras dont « le propre [...] est de tenir les esprits, les yeux et les oreilles dans un égal enchantement » (La Bruyère 2011 : 92). 
littéraires, demeure encore passablement ignoré. Je souhaiterais lui accorder maintenant le premier rôle.

Si le XVII e siècle persiste à croire - ou feint de croire - aux pouvoirs magiques d'un œil qu'il considère encore comme le reflet présumé de l'âme : «Et je vois dans les yeux », prévient le Pacorus de Corneille, "ce qui se passe en l'âme » (Suréna, V, 3) ${ }^{8}$, les adeptes de la nouvelle science refusent d'entériner la pertinence cognitive du regard. Dénouant l'antique rapport de connivence entre l'œil et le monde, dans lequel le second agissait sur le premier en lui conférant une partie de sa nature, le regard baroque perd du même coup l'assurance que ce qui lui parvient est la manifestation de la vérité. Les travaux de Kepler et de Descartes sont sur ce point aussi catégoriques qu'emblématiques : la relation directe que l'on estimait jusqu'alors indiscutable entre l'œil et le monde doit être repensée à nouveaux frais. Entre les sens et le sensible, le rapport n'est plus d'identité, il n'est que de signifiants à signifiés. L'œil ne saisit plus du monde que des signes et, selon Descartes, la confiance que l'on doit accorder aux sens ne saurait dépasser celle permettant de distinguer ce qui peut être physiquement utile ou nuisible. Ainsi, l'évidence sensible de chaleur ou de douleur occasionnée par le feu ne persuade pas Descartes « qu'il y a dans le feu quelque chose de semblable à cette chaleur, non plus qu'à cette douleur ; mais seulement [...] quelque chose en lui [...] qui excite en moi ces sentiments de chaleur ou de douleur» (Descartes $1967: 6,485$ ). De même, il n'y a « aucune ressemblance entre la couleur que nous supposons être en [un] objet et celle qui est en notre sens » (Descartes $1973: 1$, $\$ 70,58$ ). La couleur est une modalité de la matière, alors que sa compréhension, comme celle de la chaleur ou de la douleur, est une modalité d'intelligibilité. Il peut y avoir une certaine analogie métaphorique entre ces divers modes, il n'y a pas homologie. Cela va bien entendu contribuer à cette «crise du sensible » que connaît la première modernité et au cours de laquelle la vision traverse une phase capitale de son histoire?

Jusqu'à l'épistémè baroque, le caractère véridique de la perception résidait dans l'absence de toute réflexion ou réfraction pouvant fausser le parcours des rayons visuels émanant des choses (intussusceptio) ou, comme certains le croyaient encore malgré les conclusions pourtant incontestables de Kepler $^{10}$, provenant de l'œil (extramissio). Mais, quelle que fût la théorie concernant

8 Plus récemment, lors d'une conférence de presse (16 juin 2001) à l'occasion d'une rencontre en Slovénie avec Vladimir Poutine, George W. Bush déclara le plus sérieusement du monde : «I looked the man in the eye. I was able to get a sense of his soul ». La Folie, mise en scène par Érasme, considérait elle aussi le regard comme « le miroir de l'âme le moins menteur» (Érasme $1964: 19$ ).

9 La « crise du sensible » fut initiée par Copernic dont l'un des mérites, selon Kant, fut de sêtre " risqué, d'une manière contraire aux sens, mais pourtant vraie, à chercher l'explication des mouvements observés non dans les objets du ciel, mais dans leur observateur » (Kant 1980 : 49). Copernic avance une représentation de l'univers allant à l'encontre d'une cosmologie validée par l'expérience apparemment indubitable des sens et par conséquent, croyait-on, par la raison naturelle. En problématisant la relation entre perception et réalité, Copernic conteste ce qui était à l'origine de l'erreur d'Aristote : l'identification de la vérité avec l'apparence sensible, dont découlent, entre autres pernicieuses "évidences", le géocentrisme et l'immobilité terrestre.

10 C'est à Porta et à son artifice de la chambre noire que Kepler attribue l'honneur d'avoir finalement tranché la question : "C’est donc une querelle terminée que celle de savoir si la vision se fait par réception ou par émission ! (Kepler 1980 : 374). Nonobstant, Descartes croit encore au rayon visuel émanant, contrairement à "l'ordinaire des hommes ", de l'œil des chats et autres félins (Descartes $1996: 1,86$ ). 
la « question tant controversée de l’intromission de la clarté ou de la lumière » (Porta 1913 : 280), c'était toujours en pleine présence que le monde rencontrait l'œil, ou que celui-ci rencontrait le monde. Savoir consistait alors à commenter ce que l'on voyait, à recueillir la prolifération des signes déposés à cette intention sur les choses et les êtres. C'est cette forme d'absolue coïncidence avec le monde sensible, ce rapport immédiat et supposé ressemblant, que la science optique de la première modernité fait voler en éclats. À une perception présumée naturelle et analogique de l'image va se substituer une interprétation sémiotique, donc culturelle, de l'indice.

À cette mise en question d'une similitude jusqu'alors indiscutable et indiscutée entre le monde et sa perception oculaire s'ajoute le fait, qu'après Galilée, l'œil ne saurait appréhender directement la nature. Celle-ci n'est plus donnée à travers tout un jeu de correspondances signalées par d'opportunes « signatures » déposées à la surface des choses mais, écrite " dans la langue mathématique» (Galilée 1980 : 141), elle demande à être savamment construite. Ne relevant plus d'une simple saisie empirique, la connaissance du monde ne résulte plus de la sensation immédiate, mais dépend d'une traduction mathématique purement théorique, i. e. imperméable aux sens. Du fait de la mathématisation ou, pour mieux dire, de la géométrisation du réel, la connaissance empirique échappe de la sorte à l'horizon des sens naturels, et en particulier à celui de la vue, et se trouve subordonnée à une minutieuse vérification expérimentale ; une expérimentation, telle que l'envisage par exemple Bacon ${ }^{11}$, qui n'a plus rien de commun avec cette "expérience vague " des phénomènes, partielle et non fondée en raison, que rejette Spinoza ${ }^{12}$. Ainsi, afin de faire advenir la vérité du monde, il conviendra de la soustraire à l'œil de chair, considéré jusqu’alors comme un irréfutable témoin, et de la soumettre désormais à celui de l'esprit.

En dénonçant un système analogique rassurant où le signe se confondait avec ce qu'il représentait $^{13}$, la science moderne, en parfaite harmonie avec l'émergence de la science optique, ruine le paradigme des correspondances présidant à la disposition en miroir du macrocosme et du microcosme qui, jusqu'ici, avait tissé un lien étroit de parenté entre les sens et le sensible. L'empire des signes l'emportant désormais sur l'empire des sens, l'avènement d'une sémiotique totalement affranchie de tout lien de ressemblance avec ce qu'elle donne à voir ne pouvait que modifier l'accès au visible.

Si, dans l'histoire de la vision, il existe une discontinuité entre le Moyen Âge et la première modernité, cette coupure ne réside pas, comme l'affirmait Lucien Febvre, dans un prétendu avènement de l'œil (Vuillemin 2013 : 235 sqq), mais bien davantage dans la mise en cause des aptitudes jusqu’alors reconnues à celui-ci. Les yeux, ces « lunettes taillées de la main de Dieu » (Arnauld et Nicole 1992 : 276), continuent à s'auréoler d'une collusion avec le divin, à bénéficier de la symbolisation positive d'une justice et d'un pouvoir politique infaillibles ${ }^{14}$, et à jouir de leurs facultés es-

11 Bacon propose de remplacer l'experientia, i. e. l'expérience commune, par un experimentum, c'est-à-dire par une "expérimentation" que les sens, ayant "quelque chose de faible et d'égarant ", ne pourraient seuls mener à bien (Bacon 1986: 116).

12 La perception acquise par l'« expérience vague », prévient Spinoza, " est tout à fait incertaine et interminable » (Spinoza $1996: 21)$.

13 Exit à jamais la représentation anthropocentrique de l'univers ptolémaïque clos et hiérarchisé, justifié par la théorie aristotélicienne des "lieux naturels" et unifié par un système absolument réflexif d’analogies et de similitudes qui, Foucault l'a admirablement décrit, s'enroulait sur lui-même (Foucault 1966 : 32-33).

14 «Like God and the perfect magistrate, the eyes saw and comprehended all things. Watchful looking was already the analogous of vigilant justice and social control, long before the advent of Jeremy Bentham's Panopticon » (Clark 2007 : 12). 
thétiques, mais la science leur dénie maintenant leurs prérogatives cognitives. L'œil cesse d'être l'outil idoine de la connaissance. Dans le champ de la galanterie, ce que disent les yeux a beau l'emporter encore souvent sur ce que disent les mots et, croit-on, livrer les secrets des passions de l'âme, dans le domaine scientifique on sait qu'il faut désormais se défier de leurs témoignages et qu'il est capital de distinguer les yeux de la perception des yeux de la connaissance ${ }^{15}$. Comme le met continument en scène son théâtre, la première modernité découvre en effet que le plaisir de voir ne rime plus avec la curiosité de savoir, et que ce que l'on tenait jusqu'alors pour la meilleure façon de connaître le monde n'est en définitive que le plus sûr moyen de (se) tromper. « [L]e plus grand obstacle et le plus grand égarement de l'entendement humain ", décrète à ce sujet Bacon, " provient de l'hébétude, de la grossièreté et des déceptions des sens » (Bacon 1986 : 115). Afin de parvenir à la connaissance, l'individu doit par conséquent trouver le moyen de négocier avec le " temps des sens trompeurs", pour reprendre l'expression de Foucault, et en particulier avec la vue, « de tous les sens, le plus facile à décevoir » (Scudéry 2004 : 368).

La vision ne recouvrera une partie de sa pertinence cognitive que lorsqu'on aura accepté, du moins chez les savants, qu'elle n'est plus immédiatement adéquate au visible, et que l'on sera persuadé que l'accès aux choses ne peut se faire que de façon métaphysique, c'est-à-dire que " nous les concevons par la pensée " (Descartes 1967 : 2, 429). C’est par le jugement de l'esprit, et non par le rapport des sens, que les choses sont convenablement perçues. Fort de ce principe de la science moderne, Galilée peut ridiculiser le simpliste Simplicio, le sectateur de la scientia aristotélicienne, qui demeure persuadé que « les expériences sensibles [doivent] l'emporter sur tout raisonnement fabriqué par l'esprit humain » et prétend que son maître à penser voulait « que ceux qui nient le témoignage du sens [soient] châtiés en perdant ce sens » (Galilée 1992 : 125).

Dans cette « crise du sensible », que l'on aurait d'ailleurs mieux fait de qualifier de « mutation du sensible » étant donné qu'elle marqua moins le développement salutaire ou fatal d'une pathologie qu'elle inaugura un changement radical de paradigme, une place d'importance a été légitimement accordée à Kepler et à Descartes, deux des plus lucides artisans de la nouvelle façon de considérer la vision ${ }^{16}$. Dans l'aventure des sens et de la connaissance, le Kepler des cinq premiers chapitres de l'Astronomiae Pars Optica, isolés sous le titre de Paralipomènes à Vitellion (1604) et, subsidiairement, de la Dioptrice $(1611)^{17}$ et, une génération plus tard, le Descartes de La Dioptrique (1637), méritent indubitablement d'être rangés au nombre de ces "fondateurs de discursivité » dans l'acception qu'esquisse Foucault en conclusion de "Qu'est-ce qu'un auteur?». Ce texte de Descartes et ceux de Kepler - que Descartes considérait comme son " $1^{\text {er }}$ maître en Optique » ("Lettre à Mersenne », 31 mars 1638) - permettent en effet de promouvoir leur auteur au rang de «fondateur » ou d'« instaurateur » de discursivité en ce qu'ils se situaient moins par rapport à la science que la science allait être dorénavant contrainte de se rapporter à eux comme à des coordonnées premières.

15 «[... la Philosophie / Dit vrai, quand elle dit que les sens tromperont, » remarque La Fontaine, « Tant que sur leur rapport les hommes jugeront " (Un animal dans la Lune, p. 283). Mais, précise à juste titre le fabuliste : «[...] si l'on rectifie / L'image de l'objet sur son éloignement, / Sur le milieu qui l'environne, / Sur l'organe, et sur l'instrument, / Les sens ne tromperont personne » (ibid.).

16 Sur Kepler et Descartes, voir en particulier Simon 2003: 203-241 et Hamou 1999 et 2001: I 195-288.

17 "Ces deux publications ", écrit Isabelle Pantin, «signalent le moment où l'optique devient, sans trop d'ambiguïté, une physique de la lumière, munie d'une théorie mathématique assez élaborée pour soutenir cette ambition " (Pantin 2010 : 257). Si avec Kepler il y eut révolution plutôt quévolution, c'est que jusqu'alors l'optique était davantage la science de la vision que celle de la lumière (Smith 2010). 
Au lieu de ratiociner sur les vertus du regard et les beautés de l'œil, Kepler expose dans ses « compléments » aux écrits de Vitellion - un moine polonais du XIII siècle dont l'Optique (1270) s'inspirait du De Aspectibus d'Alhazen - un mécanisme physico-géométrique rendant compte de la réfraction atmosphérique et de la nature de la lumière. Alors que dans la tradition optique héritée d'Aristote voir consistait à être affecté par la forme du sensible actualisée par la lumière dans l'air environnant puis ensuite dans l'œil, Kepler avance la thèse que la vision est affaire d'images rétiniennes tributaires de la réfraction. À une vision reposant sur le principe d'une perception sans médiation et dans laquelle l'objet observé était lui-même présent par sa forme, mais sans sa matière, dans l'humeur cristalline grâce à son empreinte déposée par ces émanations sensibles que la tradition appelait eidos ou species, Kepler oppose une théorie novatrice. Contre la coïncidence qui existerait entre le monde et l'œil, les images rétiniennes envisagées par Kepler supposent une certaine forme de médiation : la vision ne peut advenir que si les rayons lumineux se trouvent concentrés par la lentille de l'œil. Récusant le principe d'un regard non médiatisé, et libérant la vision de sa subordination à l'astronomie et aux expériences curieuses inhérentes à la magie naturelle ${ }^{18}$, Kepler fonde ainsi l'optique moderne en lui conférant un savoir distinct et en lui assignant le champ autonome qui, dès lors, sera le sien. Dans La Dioptrique, Descartes explicite quant à lui le rôle du nerf optique dans le processus de transmission des images rétiniennes jusqu’aux instances de jugement situées dans le cerveau, sensus communis, et conteste toute nécessité de ressemblance entre «les idées que [l’âme] conçoit, et les mouvements qui causent ces idées » (Descartes $1996: 6,131)$, i. e. entre les images cérébrales et leurs référents dans le monde.

Dans ses Paralipomènes, Kepler assimile l'œil à une camera obscura, ce dispositif mécanique permettant d'observer les éclipses à loisir, et sans risque pour l'œil ${ }^{19}$. En s'appliquant à faire la théorie de cette chambre noire afin de résoudre les erreurs de mesure résultant d'une mauvaise compréhension des conditions de la vue $e^{20}$, non seulement fait-il passer dans le domaine de la science un dispositif appartenant jusqu'alors au domaine de la magie naturelle, mais il est également en mesure d'ébaucher une théorie révolutionnaire du processus de la vision dans lequel la réfraction, je l'ai dit, joue un rôle capital. Ce faisant, il inscrit dans le champ de la physiologie et de la physique un processus qui relevait jusqu’alors de la psychologie ou, pour le dire de façon non anachronique, qui avait partie liée avec le travail de l'âme.

S'il y a production d'images à l'intérieur de cette chambre noire qu'est l'œil, celles-ci ne correspondent pas à un double sensible des objets vus, mais relèvent au contraire d'une relation mécaniste entre "l'objet visible » et sa " peinture sur la paroi blanche et concave de la rétine " (Kepler 1980 : 319). Comme une vulgaire chambre noire, l'œil possède un petit orifice : la pupille, faisant office de diaphragme ; un cristallin, jouant le rôle de «lentille convexe en forme d'hyper-

18 La magie naturelle consistait à montrer des phénomènes naturels propres à surprendre et à émerveiller. Les traités de magie naturelle sont nombreux au cours du XVI siècle et l'un des plus célèbres fut celui, déjà cité, de Porta, Magia Naturalis (Naples, 1558), édité et réédité sans cesse jusqu’au second tiers du XVII siècle.

19 Pour les rapports entre la chambre noire et la peinture, notamment celle de Vermeer, voir les belles réflexions de Jacques Darriulat, "Le mystère de la chambre noire " (Darriulat et Enthoven 2011 : 173-230). Si la chambre noire est une « machine voyante », l'œil est pour Darriulat « une chambre noire douée de conscience » (Darriulat et Enthoven 2011 : 202).

20 Il résolut en particulier l'énigme des dimensions réduites du disque lunaire occultant le soleil tel qu’il apparaissait dans la chambre noire et qui ne correspondait pas à ce que donnait à voir l'œil. Lanalyse subséquente de la réfraction lui donna la possibilité d'arriver, dans la Dioptrice, à une théorisation et à une légitimation des lentilles fabriquées jusque-là de manière fort hasardeuse. 
bole» (Kepler 1990 : 52); et un écran : la rétine. Tenu à tort par la tradition pour l'organe sensitif responsable de la transmutation du visible en psychique, la fonction de l'humeur cristalline acquiert chez Kepler celle d'une lentille interne dont le rôle de dioptre convergent, à l'instar de ce que feraient « au hasard d'expérimentations » diverses des «boules de cristal » ou des « urinals de verre remplis d'eau claire» (Kepler $1980: 333$ ), rend la " peinture » rétinienne aussi nette que possible. Ce qui s'inscrit sur la rétine, et non sur la choroïde ou sur le cristallin, est donc une image stigmatique réelle que Kepler conçoit, non comme une simple projection de la pupille, mais comme le résultat d'une "passion pénétrante» (Kepler 1990 : 53) donnant lieu à une espèce de gravure résultant d'une petite combustion de la lumière sur la rétine. Il s'agit donc d'une pictura, c'est-à-dire d'une entité matérielle tangible, distincte à la fois des simulacres postulés par la tradition $^{21}$, et d'une imago, c'est-à-dire d'une image mentale fantasmatique flottant quelque part devant les yeux ou perçue dans un miroir ou sur une surface réfringente ${ }^{22}$.

Il restait toutefois à savoir comment ces picturae pouvaient se transmuer en sensations et/ou en pensées. Kepler ne se prononce pas et laisse aux " Physiciens ", c'est-à-dire aux médecins et autres anatomistes, le « soin d'en disserter» (Kepler 1980 : 317). Le problème était complexe : comment est-ce qu'une image optique concrète, dessinée par des faisceaux de points lumineux et colorés au fond des yeux, autour des conduits obscurs et tortueux du nerf optique - que l'on croyait, aux dires de Galien, creux et dans l'axe de l'œil -, pouvait-elle être vue et transmise ensuite au cerveau, «tribunal de l'âme » (Kepler 1980 : 317) ? Sept ans plus tard, dans la Dioptrice, Kepler déclare encore que la manière dont cette transmission se fait " est d'une raison cachée " (Kepler 1990 : 54). L'énigme était incontestablement délicate à résoudre et le nouveau modus visionis de Kepler conduisait à supposer diverses hypothèses, dont celle, traditionnelle et suggérée dans la Dioptrice, de la présence dans le nerf optique d'un " esprit voyant " (Kepler 1990 : 53) s'imprégnant de l'image rétinienne puis l'acheminant jusqu'au cerveau. Outre la possibilité d'une transmission immatérielle et instantanée, on pouvait également supposer un œil second à l'intérieur du cerveau capable de percevoir la peinture rétinienne... C’est ce que récusera Descartes (Descartes $1996: 6,130$ ) en soustrayant le processus de la vision aux lois traditionnelles de l'optique et, à la suite de Kepler, en le soumettant à celles de la physiologie. Mais, et là réside l'un des aspects de la nouveauté, il dote ce processus de deux moments distincts : une phase nerveuse relevant du corps et une phase mentale dépendant de l'«âme ", i. e. du jugement. La seconde et déterminante innovation qui va permettre à Descartes de résoudre le problème soulevé par Kepler sera de rejeter l'idée apparemment évidente que ce qui parvient au cerveau est une réplique ressemblante du visible.

Souscrivant à l'explication dioptrique des images rétiniennes proposée par Kepler, Descartes postule que celles-ci ne constituent qu'un moment d'un processus qui se poursuit dans le corps opaque du cerveau où se peignent d'autres picturae physiologiques dépourvues de qualité optique et ne produisant aucune imago. Passant d'un modèle iconique à un modèle sémiotique, il propose une description mécanique de la vision selon laquelle les "petits filets du nerf optique » (Descartes 1996 : 6, 131), à la manière d'un bâton d'aveugle transmettant une pression de l'une

21 Selon Démocrite et Épicure, la vision serait le fait de fines particules qui, entre l'objet et l'œil, formeraient des simulacra (eidôla), c'est-à-dire des répliques de l'objet transportant ses caractéristiques jusqu’à l'œil.

22 C'est de "toutes ces petites images voltigeantes par l'air, nommées des espèces intentionnelles " que Descartes souhaite délivrer l'esprit de ses lecteurs (Descartes $1996: 1,85$ ). 
de ses extrémités à l'autre, propagent jusqu'au cerveau les images rétiniennes ${ }^{23}$. S’il existe une certaine ressemblance physique entre l'image rétinienne et l'objet extérieur, cette ressemblance disparaît ensuite entre l'image rétinienne et l'image cérébrale. Ce qui parvient au cerveau, loin de transférer les ressemblances « assez parfaites dans le fonds de nos yeux » des « objets que nous regardons " (Descartes $1996: 5,114$ ), ne relève plus que d'un processus mécanique mettant en œuvre une configuration d'étendue et de mouvements apte à être décodée par l'entendement. En plus de l'analogie avec le type de perception qui permet à un aveugle de " voir » le monde à l'aide d'un simple bâton transformant le sensible en visible, Descartes évoque, bien avant Saussure et Magritte, la nature arbitraire du signe linguistique ${ }^{24}$, et allègue la sémiotique des gravures en taille-douce pour prouver que la perception, loin d'être corrélée à la ressemblance, est au contraire affaire de représentation. Ce qui cause la perception n'est pas l'objet lui-même, ni une reproduction lui ressemblant, mais un substitut de cet objet qui, tel le signe linguistique, doit être moins perçu que décrypté.

Contrairement à Leibniz qui estime encore qu'il existe une " manière de ressemblance [...] expressive » entre les « idées [et] leurs causes » (Leibniz 1966 : 109), Descartes affirme que le fonctionnement de la vision réside dans les idées du visible que l'âme déchiffre à partir des signes inscrits dans le cerveau. Signes qui ne sont représentatifs que parce qu'ils sont distincts de leurs référents et, paradoxe apparent, d'autant plus parfaits qu'ils leur sont dissemblables. Comme les gravures en taille-douce qui, «n'étant faites que d'un peu d'encre posée çà et là sur du papier, nous représentent des forêts, des villes, des hommes, et même des batailles et des tempêtes » (Descartes 1996:4, 113), les images qui parviennent au cerveau, « pour être plus parfaites [...] et représenter mieux un objet, elles doivent ne lui pas ressembler» (Descartes 1996: 4, 113). Fonctionnant par analogie avec « un peu d'encre » ou des " paroles ", l'acte de voir repose sur des signes se bornant à indiquer une propriété dont ils ne véhiculent pas la ressemblance objective. Ce processus sémiotique élémentaire éclaire la vérité d'une vision qui ne donne pas un accès direct au réel, mais seulement une image infidèle de celui-ci. Ne possédant en lui aucune réplique sensorielle de l’objet, le sujet ne peut donc pas se fier à ses sens pour connaître ce qui l'affecte : «c'est l'âme qui voit », conclut Descartes, « et non pas l'œil » (Descartes 1996 : 6, 141). C’est précisément cela que ne comprennent pas les personnages abusés du théâtre de l'époque : relevant du raisonnement et non directement de la sensation, le monde objectif n'est plus celui que sent ou voit le sujet.

C'est parce qu'il sait que sa blonde Marquise « n'a nulle teinture de science » (Fontenelle 1998 : 51), et qu'il subodore vraisemblablement qu'elle n'est pas une spectatrice assidue du théâtre du temps, que le galant Philosophe des Entretiens sur la pluralité des mondes de Fontenelle peut espérer briller à peu de frais : " Toute la philosophie », pontifie-t-il, «n'est fondée que sur deux

23 C'est à travers un processus mécanique similaire que se formeraient les taches de naissance : l'image rétinienne peut quelquefois transiter " par les artères d'une femme enceinte, jusques à quelque membre déterminé de l'enfant qu'elle porte en ses entrailles» (Descartes $1996: 5,129$ ).

24 «[... il y a plusieurs autres choses que des images, qui peuvent exciter notre pensée ; comme, par exemple, les signes et les paroles, qui ne ressemblent en aucune façon aux choses qu’elles signifient » (Descartes 1996: 4, 112). Idée déjà formulée, mais non encore publiée, au début du Monde. 
choses, sur ce qu'on a l'esprit curieux et les yeux mauvais » (Fontenelle 1998 : I 62). En 1686, le scoop n'en est pourtant plus un ou, comme aurait pu rétorquer le prince Ladislas de Rotrou : "Ce mystère, ma sœur, n'est plus une nouvelle » (Venceslas, II, 3). À cette date, il est désormais parfaitement établi que la "Philosophie », i. e. la science, résulte d'un état de fait inversement proportionnel à la capacité sensorielle de la vue, le sens qui passait jusqu'alors pour contribuer le plus à la connaissance. La première modernité se révèle à cet égard épistémologiquement révolutionnaire : ce n'est plus parce que nous voyons bien que nous philosophons mais, comme le résume Fontenelle, c'est au contraire parce que nous voyons mal. Et c'est dans l'espoir de combler cette concupiscentia oculorum, ce désir forcené de voir fustigé par saint Augustin (Augustin 1964), cette " concupiscence des yeux » (Pascal 2004 : fr. 460), ou encore cette pulsion scopique qu'analysera Freud (Freud 2012: 83 sqq.) que « l'œil de la raison » (Rotrou, Céliane, IV, 1) ne peut plus se satisfaire de ce que lui offre l'œil de chair qui, pour emprunter à Clorimand, un autre personnage de Rotrou, "pensant beaucoup voir, en effet ne voit pas » (Les Occasions perdues, II, 3).

La première modernité découvre qu'il est impératif de savoir dénouer le témoignage des sens du raisonnement de l'esprit afin de ne pas ériger le premier en principe de connaissance du second. Abducere mentem a sensibus. Une fois cette précaution prise, on pourra spéculer avec bonheur sur les apparences. C'est en particulier ce qu'enseigne l'exemple cartésien du morceau de cire dont l'appréhension par les sens s'avère tout à fait inefficace pour en dévoiler la nature essentielle (Descartes 1967 : 2, 423 sqq.). Sous ses apparences changeantes d'odeur, de couleur et de saveur, la cire demeurerait à jamais inconnaissable si elle ne dépendait que des catégories du sensible. Le monde physique, afin d'être véritablement connu, doit être désormais conçu et non plus seulement senti : sa perception légitime ne doit désormais résulter que de l'« entendement seul» (Descartes 1967 : 2, 425). La démonstration est également probante en ce qui concerne ces " hommes qui passent dans la rue " (Descartes $1967: 2$, 426) et que le philosophe regarde depuis sa fenêtre. Que voit-il réellement, "sinon des chapeaux et des manteaux, qui peuvent couvrir des spectres ou des hommes feints qui ne se remuent que par ressorts ? (Descartes $1967: 2$, 427). Les hommes que Descartes voit de ses yeux, du haut de sa fenêtre, ne sont peut-être que des « hommes feints ", mais ceux qu'il voit «par la seule puissance de juger qui réside en [son] esprit » sont eux en revanche de « vrais hommes» (Descartes $1967: 2,427$ ).

Ce n'est par conséquent que par un raccourci audacieux que l'on dit voir des hommes, comme précédemment l'on voyait un morceau de cire. Au lieu de dépendre de la vision des yeux, une connaissance digne de ce nom doit résulter d'une opération de l'esprit, d'un jugement purement intellectuel. Comme la connaissance de la cire, la réalité de ces hommes est tributaire de la « seule inspection de l'esprit » (Descartes $1967: 2,426$ ) ; elle passe par « la faculté d'entendre qui est en nous, et non point par l'imagination ni par les sens » (Descartes 1967 : 2, 429). Révolution épistémologique qui, tout en continuant à faire de la vue, comme l'écrira encore Malebranche vers la fin du XVII e siècle, "le premier, le plus noble et le plus étendu de tous les sens ${ }^{25}$ ", va octroyer aux " yeux de l'esprit » (Rotrou, Célie, III, 2), ces « oreilles de l'âme » comme les appelle Théodore dans Bélisaire (IV, 6), une prééminence incontestable sur, et l'expression est récurrente chez Rotrou et

25 Malebranche 1979 : I 54. Comme Platon, qui met en garde contre l'attachement au monde sensible, Malebranche a beau louer les beautés de l'œil, le sectateur de Descartes reproche lui aussi de juger « de la vérité des choses par l'impression sensible qui nous trompe toujours » et de négliger « les idées claires et distinctes de l'esprit, qui ne nous trompent jamais » (Malebranche 1979 : I 136). 
ses confrères dramaturges, les « yeux éblouis » du corps. Et ce n'est évidemment pas parce que Descartes considérait la vue comme « le plus universel et le plus noble » de nos sens (Descartes 1996:1, 81) qu'il était prêt à lui conférer un privilège cognitif supérieur à celui des autres sens.

À cet égard, et sur un mode quelque peu décalé, une scène similaire des Sosies de Rotrou (1636) et de l'Amphitryon de Molière (1668) est instructive. Il s'agit de l'épisode des trois capitaines que l'on a fait venir afin de mettre au jour l'imposture en distinguant le véritable Amphitryon de celui qui a emprunté son identité et couché avec sa femme. Les arbitres improvisés s'avèrent bien entendu incapables de différencier grâce à leurs yeux le général thébain de son usurpateur, et doivent s'en remettre au regard de leur ventre : «Point, point d'Amphitryon où l'on ne dîne point » décrète un capitaine chez Rotrou (IV, 4) ; " Le véritable Amphitryon ", déclare à son tour le capitaine de Molière, «Est l'Amphitryon, où l'on dîne » (III, 5). Si la vérité, ou du moins ce que l'on prend pour telle, ne résulte pas des sensations mais de l'entendement de l'esprit (ou du ventre), l'on doit par conséquent admettre que l'individu qui raisonne (qui panse ?) voit mieux, et beaucoup plus loin, que celui qui ne fait que regarder. Dans le même ordre d'idées, l'on remarquera que l'extrême efficacité des fréquents travestissements de la scène baroque, en plus des effets de surprise, des rebondissements dramatiques et de l'érotisme ambigu qu'ils peuvent parfois susciter, rappellent aussi sans cesse aux spectatrices et spectateurs du text scénique les limites d'une acuité visuelle inapte à déceler l'identité véritable de celui ou de celle qui se cache sous de bien improbables déguisements. Aveugles-voyants incapables de discerner la personne du personnage, ces protagonistes, avatars de ceux de l'univers de la caverne platonicienne, ne voient pas au-delà des apparences et des simulacres de la réalité sensible.

$\mathrm{Si}$, comme le prétend Foucault, la première modernité, réfractaire aux éventuels enseignements de la folie, contraignit les corps en faisant en sorte que l'enfermement fasse suite à de supposées déportations loin des rives de la raison, elle présida aussi à cet autre enfermement dont ne parle pas l'auteur de l'Histoire de la folie : l'asservissement de l'œil fasciné et fascinant à la réflexion normée et analytique du regard raisonnable. Au primat aristotélicien du sensible succède le règne de l'intelligible. Pour que le plaisir de voir renoue avec la curiosité de savoir, les nouveaux chemins de la connaissance exigent que la vision se fasse désormais avec les yeux de l'esprit, non avec ceux du corps. Il est de ce fait logique que Descartes, après avoir affirmé « la fausseté ou l'incertitude qui se trouve en tous les jugements qui dépendent du sens ou de l'imagination ", conclue à la solidité indubitable des jugements « qui ne dépendent que de l'entendement pur $^{26}$ ».

Au moment où la science moderne problématise la relation de l'individu aux réalités qui l'entourent, le théâtre baroque problématise la relation de ses personnages à leur univers scénique ainsi que la relation des véritables spectateurs à la représentation dramatique. Dans le champ scientifique comme sur la scène du théâtre, ce regard nouveau caractéristique de l'épistémè baroque est marqué par l'abandon du préjugé anthropocentrique qui considérait les yeux comme le critère absolu de connaissance et de vérité. Contrairement à ce que pouvait penser Socrate, qui

26 «Lettre à Mersenne », mars 1637. Si pour Descartes, « [1]'entendement seul [...] a le pouvoir de percevoir la vérité », il n’en demeure pas moins que celui-ci « doit pourtant se faire aider par l'imagination, les sens et la mémoire afin de ne rien négliger de ce qui fait partie de nos ressources » (Descartes 1963: 12, 135). Jetant un pont entre l'intellect et le monde, les sens et l'imagination, qui « résident et se rencontrent certainement en [nous] (Descartes $1967: 3,40$ ), ont beau être une source avérée d’erreurs, ils évitent toutefois à l'esprit de s’abîmer dans une seule activité conceptuelle. Après avoir posé la dualité âmecorps, Descartes est néanmoins convaincu que la pensée et l'étendue, constituant un tout, ne sauraient être séparées l'une de l'autre ; c'est notamment ce qui permet d'expliquer ces données mentales immédiates que sont les couleurs et les douleurs. 
disait au bel Alcibiade que « La vision de l'esprit ne commence à être perçante, que quand celle des yeux commence à perdre de son acuité » (Platon 1998 : 171), la première modernité apporte la preuve que l'âge n'a rien à voir à la chose et pour que le plaisir de voir renoue avec la curiosité du savoir, il est impératif que la vision se fasse avec l'œil de l'esprit, qui ne puise qu'en lui-même, et non plus avec celui du corps, qui doit tout à la perception. Emblématique de la première modernité dont il illustre admirablement l'une de ses prémices scientifiques essentielles, le théâtre baroque plaide pour une vision qui va au-delà du visible ; d'un voir qui, excédant la vue, rimera à nouveau avec savoir, mais plus encore avec pouvoir.

\section{Références bibliographiques}

Aristote. Métaphysique. (1991). J. Tricot (Ed.). Paris: Vrin.

Arnauld, A.; \& Nicole, P. (1992 [1662]). La Logique ou L'Art de penser. Ch. Jourdain (Ed.). Paris: Gallimard. Augustin. (1964). Les Confessions. J. Trabucco (Ed.). Paris: GF Flammarion.

Bacon, F. (1986 [1620]). Novum Organum. M. Malherbe, \& J.-M. Pousseur (Eds.). Paris: PUF.

Barthes, R. (2002). Le démon de l'analogie. In Roland Barthes par Roland Barthes. E. Marty (Ed.), Roland Barthes, Euvres complètes (vol. 4) (p. 624). Paris: Seuil.

Clark, S. (2007). Vanities of the Eye. Vision in Early Modern European Culture. New York: Oxford University Press.

Corneille, P. (1980-87). CEuvres complètes. G. Couton (Ed.). 3 vol. Paris: Gallimard.

Darriulat, J.; \& Enthoven, R. (2017). Vermeer. Le jour et l'heure. Paris: Fayard.

Descartes, R. (1963). Règles pour la direction de l'esprit. In F. Alquier (Ed.), Descartes Euvres philosophiques (vol. 1) (pp. 67-204). Paris: Garnier.

. (1967). Les Méditations métaphysiques. In F. Alquier (Ed.), Descartes Euvres philosophiques

(vol. 2) (pp. 375-505). Paris: Garnier.

. (1973). Les Principes de la philosophie. In F. Alquier (Ed.), Descartes Cuvres philosophiques (vol.

3) (pp. 83-525). Paris: Garnier.

. (1996). La Dioptrique. In Ch. Adam, \& P. Tannery (Eds.), Euvres de Descartes (vol. 6) (pp. 78228). Paris: Vrin.

Érasme. (1964 [1511]). Éloge de la folie. P. de Nolhac (Trad.). Paris: GF Flammarion.

Fontenelle, B. (1998 [1686]). Entretiens sur la pluralité des mondes. Ch. Martin (Ed.). Paris: GF Flammarion.

Foucault, M. (1966). Les Mots et les choses. Une archéologie des sciences humaines. Paris: Gallimard. (1969). L’Archéologie du savoir. Paris: Gallimard.

. (1976). Histoire de la folie à l'âge classique. Paris: Gallimard.

(1994 [1969]). Qu'est-ce qu'un auteur ? In D. Defert, \& F. Ewald (Eds.), Dits et Écrits, 1954-1988

(vol. 1) (pp. 789-821). Paris: Gallimard.

Freud, S. (2012 [1915]). Pulsions et destins des pulsions. O. Mannoni (Trad.). Paris: Payot \& Rivages.

Galilée. (1980). L’Essayeur de Galilée. 1623. Ch. Chauviré (Trad.). Paris: Les Belles Lettres.

. (1992 [1633]). Dialogue sur les deux grands systèmes du monde. R. Fréreux, \& F. De Gandt

(Trads.) Paris: Seuil.

Gougenot, N. (2000 [1633]). La Comédie des comédiens. F. Lasserre (Ed.). Tübingen: G. Narr Verlag.

Hamou, P. (1999 et 2001). La Mutation du visible. Essai sur la portée épistémologique des instruments d'optique au XVII siècle. 2 vol. Villeneuve d'Ascq: Presses universitaires du Septentrion. 
Kant, E. (1980 [1787]). Critique de la raison pure. A. J.-L. Delamarre, \& F. Marty (Trads). Paris: Gallimard. Kepler, J. (1980 [1604]). Paralipomènes à Vitellion. C. Chevalley (Trad.). Paris: Vrin. . (1990 [1611]). Dioptrice. J. Peyroux (Trad.). Paris: A. Blanchard.

La Bruyère, J. de. (2011 [1696]). Des ouvrages de l'esprit. In R. Pignarre (Ed.), Les Caractères ou Les Mours de ce siècle (pp. 81-100). Paris: GF-Flammarion.

La Fontaine, J. de. (1958 [1678]). Un animal dans la lune. In P. Clarac, \& J.-P. Collinet (Eds.), Euvres complètes. Fables, contes et nouvelles (vol. 1) (pp. 282-284). Paris: Gallimard.

Leibniz, G. (1966 [1765]). Nouveaux essais sur l'entendement humain. J. Brunschwig (Ed.). Paris: Garnier-Flammarion.

Malebranche, N. (1979 [1674-1675]). De la recherche de la vérité. G. Rodis-Lewis (Ed.). 2 vol. Paris: Gallimard. Molière. (2010). CEuvres complètes. G. Forestier (Ed.). 2 vol. Paris: Gallimard.

Montaigne, M. de. (2004). Les Essais. P. Villey, \& V-L. Saulnier (Eds.). Paris: Presses universitaires de France.

Pantin, I. (2010). Species et pyramides. L'optique traditionnelle et ses impasses au temps des premiers instruments d'optique. In M. Hochmann, \& D. Jaccquart (Eds.), Lumière et vision dans les arts. De l'Antiquité au XVII siècle (pp. 257-281). Genève: Droz.

Pascal. B. (2004). Les Provinciales, Pensées et opuscules divers. Éd. G. Ferreyrolles, \& Ph. Sellier (Eds.). Paris: Librairie Générale Française.

Platon. (1998). Le Banquet. L. Brisson (Trad.). Paris GF Flammarion.

Porta, G. della. (1913 [1631]). La Magie naturelle ou Les Secrets et miracles de la Nature. Paris: H. Daragon.

Poussin, N. (1964 [1641]). À Sublet de Noyers. In A. Blunt (Ed.), Nicolas Poussin, Lettres et propos sur l'art (pp. 60-65). Paris: Hermann.

Quignard. P. (2017). Performances de ténèbres. Paris: Galilée.

Rotrou, J. de. (1998-2019). Théâtre complet. G. Forestier (Dir.). 13 vol. Paris: SDTF-Les Belles Lettres. Scudéry, G. de. (1975 [1635]). La Comédie des comédiens. J. Crow (Ed.). Exeter: University of Exeter Press.

- (2004 [1637]). Observations sur Le Cid. In J.-M. Civardi (Ed.), La Querelle du Cid (1637-1638) (pp. 347-431). Paris: Champion.

Shea, W. (2000). Looking at the Moon as Another Earth: Terrestrial Analogies and Seventeenth-Century Telescopes. In F. Hallyn (Ed.), Metaphor and Analogy in the Sciences (pp. 83-103). Dordrecht: Kluwer Academic Publishers.

Simon, G. (2003). Archéologie de la vision. L’optique, le corps, la peinture. Paris: Seuil.

Smith, M. A. (2010). Bringing the Scientific Revolution into Focus: The Case for Optics. In M. Hochmann, \& D. Jaccquart (Eds.), Lumière et vision dans les arts. De l'Antiquité au XVII' siècle (pp. 163-186). Genève: Droz.

Spinoza, B. de. (1996 [1665-1670]). Traité de la réforme de l'entendement [...].. S. Auffret (Trad.). Paris: Mille et une nuits.

Vuillemin, J.-Cl. (2001). Illusions comiques et dramaturgie baroque : Corneille, Rotrou et quelques autres, Papers on French Seventeenth-Century Literature, 28, 307-325.

. (2007). Jeux de théâtre et enjeu du regard dans le théâtre de Rotrou, Littératures classiques, 63, 239-249.

. (2013). Épistémè Baroque: le mot et la chose. Paris: Hermann. 